\title{
ONE-DIMENSIONAL DIFFUSION PROCESSES \\ IN HALF-BOUNDED DOMAINS WITH REFLECTION AND A POSSIBLE JUMP-LIKE EXIT FROM A MOVING BOUNDARY
}

\author{
Bohdan Kopytko ${ }^{1}$, Roman Shevchuk ${ }^{2}$ \\ ${ }^{I}$ Institute of Mathematics, Czestochowa University of Technology \\ Częstochowa, Poland \\ ${ }^{2}$ Vasyl Stefanyk Precarpathian National University \\ Ivano-Frankivsk, Ukraine \\ bohdan.kopytko@im.pcz.pl,r.v.shevchuk@gmail.com \\ Received: 01 August 2016; accepted: 31 August 2016
}

\begin{abstract}
By the method of the classical potential theory, we construct the two-parameter Feller semigroup of operators associated with such a diffusion phenomenon on a half-line with a moving boundary where either a reflection or jump phenomenon occurs at a boundary point.
\end{abstract}

Keywords: diffusion process, parabolic potential, Feller-Wentzell boundary condition

\section{Introduction}

In the theory of stochastic processes while studying the diffusion processes in bounded and half-bounded domains, it occurs the situation in which the continuation of motion of a diffusion particle after it reaches the boundary of the domain is performed by jumps. The question on construction of semigroups of operators associated with the diffusion process with the property of a jump-like exit from the boundary of the domain leads to the statement of a boundary-value problem for a linear parabolic equation of the second order with a nonlocal boundary condition. Since the general form of boundary conditions for a one-dimensional (with respect to spatial variable) time-homogeneous diffusion process was established in the works of W. Feller [1] and A.D. Wentzell [2], these conditions were called Feller-Wentzell boundary conditions.

In the present paper we consider the one-dimensional parabolic boundary-value problem of Wentzell (with the combination of the derivative with respect to spatial variable and the nonlocal term) for the case of an inhomogeneous diffusion process in domain $D_{s} \equiv(X(s), \infty)$ provided the lateral boundary $X(s)$ satisfies the Hölder condition with respect to the time variable with exponent $>\frac{1}{2}$. This problem is 
stated in Section 2 and is solved there by the method of ordinary parabolic potentials. Using its solution in Section 3, we construct the two-parameter semigroup of operators $T_{s, t}, 0 \leq s<t \leq T$ ( $T>0$ fixed), associated with an inhomogeneous Feller process on the closure $\bar{D}_{s} \equiv[X(s), \infty)$ of $D_{s}$ which coincides in $D_{s}$ with the diffusion process given there and its behaviour at point $X(s)$, is determined by the Feller-Wentzell boundary condition.

Note that similar problems were considered earlier in $[3,4]$ for the case of bounded and half-bounded domains with fixed boundary points. We also mention works [5-7] where the related problems were studied by the methods of stochastic analysis.

\section{Parabolic boundary-value problem of Wentzell}

Consider on plane $(s, x)$ the set

$$
S_{t}=\left\{(s, x): 0 \leq s<t \leq T ; x \in D_{s}\right\}
$$

denoting by $\bar{S}_{t}$ the closure of $S_{t}$. Let in $S_{t}$ the parabolic equation

$$
\frac{\partial u(s, x, t)}{\partial s}+\frac{1}{2} b(s, x) \frac{\partial^{2} u(s, x, t)}{\partial x^{2}}+a(s, x) \frac{\partial u(s, x, t)}{\partial x}=0
$$

is given. We shall seek a solution $u(s, x, t)$ of equation (1) satisfying the "initial" condition

$$
\lim _{s \uparrow t} u(s, x, t)=\varphi(x), x \in \bar{D}_{s}
$$

and the Feller-Wentzell boundary condition of the form

$$
-q(s) \frac{\partial u(s, X(s), t)}{\partial x}+\int_{D_{s}}(u(s, X(s), t)-u(s, y, t)) \mu(s, d y)=0
$$

$(0 \leq s<t \leq T)$.

The main problem is to find the function $u(s, x, t)$ which belongs to $C^{1,2}\left(S_{t}\right) \cap C\left(\overline{S_{t}}\right)$ and which satisfies the equation (1) in $S_{t}$, the "initial" condition (2) and the boundary condition (3).

In the present paper, the following conditions are supposed to be satisfied:

1. The coefficients $a(s, x)$ and $b(s, x)$ are bounded on $[0, T] \times \mathbb{R}$, besides, there exist positive constants $b$ and $B$ such that $0<b \leq b(s, x) \leq B$ for all $(s, x) \in[0, T] \times \mathbb{R}$. 
2. For all $s, s_{1} \in[0, T], x, x_{1} \in \mathbb{R}$ the next inequalities hold:

$$
\begin{aligned}
& \left|a(s, x)-a\left(s_{1}, x_{1}\right)\right| \leq c\left(\left|s-s_{1}\right|^{\frac{\alpha}{2}}+\left|x-x_{1}\right|^{\alpha}\right), \\
& \left|b(s, x)-b\left(s_{1}, x_{1}\right)\right| \leq c\left(\left|s-s_{1}\right|^{\frac{\alpha}{2}}+\left|x-x_{1}\right|^{\alpha}\right),
\end{aligned}
$$

where $c$ and $\alpha$ are positive constants, $0<\alpha<1$.

3. The curve $x=X(s)$ is Hölder continuous with exponent $\frac{1+\alpha}{2}$ on $[0, T]$.

4. $\varphi \in C_{b}(\mathbb{R})$, where $C_{b}(\mathbb{R})$ denotes the Banach space of bounded continuous functions on $\mathbb{R}$ with norm $\|\varphi\|=\sup _{x \in \mathbb{R}}|\varphi(X)|$.

5. The function $q(s)$ is positive and continuous on $[0, T]$.

6. $\mu(s, \cdot)$ is the nonnegative measure on $D_{s}$ such that for any $\delta>0$

$$
\int_{D_{\delta}(X(s))}(y-X(s)) \mu(s, d y)<\infty, \int_{D_{s} \backslash D_{\delta}(X(s))} \mu(s, d y)<\infty,
$$

where $D_{\delta}(X(s))=\left\{y \in D_{s}:|y-X(s)|<\delta\right\}$ and these integrals are continuous on $[0, T]$ as functions of $s$.

Denote by $G(s, x, t, y)$ the fundamental solution of equation (1) $(0 \leq s<t \leq T$, $x \in \mathbb{R}, y \in \mathbb{R}$ ). Its existence is assured by 1), 2) (see [5, Ch. II, §2], [8, Ch. IV, $\S 11]$ ). Recall that function $G$ is nonnegative, jointly continuous, continuously differentiable with respect to $s$, twice continuously differentiable with respect to $x$ and satisfies the inequality

$$
\left|D_{s}^{r} D_{x}^{p} G(s, x, t, y)\right| \leq c(t-s)^{-\frac{1+2 r+p}{2}} e^{-h \frac{(y-x)^{2}}{(t-s)}}
$$

for all $0 \leq s<t \leq T, \quad x, y \in \mathbb{R}$, where $r$ and $p$ are the nonnegative integers such that $2 r+p \leq 2 ; D_{s}^{r}$ is the partial derivative with respect to $s$ of order $r, D_{x}^{p}$ is the partial derivative with respect to $x$ of order $p$; symbols $c$ and $h$ denotes (here and in what follows) any one of various different positive constants.

Recall also that

$$
G(s, x, t, y)=Z_{0}(s, x, t, y)+Z_{1}(s, x, t, y)
$$

where

$$
Z_{0}(s, x, t, y)=\frac{1}{\sqrt{2 \pi b(t, y)(t-s)}} e^{-\frac{(y-x)^{2}}{2 b(t, y)(t-s)}}
$$


and the function $Z_{1}$ satisfies inequality

$$
\left|D_{s}^{r} D_{x}^{p} Z_{1}(s, x, t, y)\right| \leq c(t-s)^{-\frac{1+2 r+p-\alpha}{2}} e^{-h \frac{(y-x)^{2}}{(t-s)}}
$$

where $0 \leq s<t \leq T, x, y \in \mathbb{R}, 2 r+p \leq 2, \alpha$ is the constant in 2 .

Having the fundamental solution $G$, we now define the parabolic potentials that will be used to solve the problem (1)-(3), namely the Poisson potential

$$
u_{0}(s, x, t)=\int_{\mathbb{R}} G(s, x, t, y) \varphi(y) d y, 0 \leq s<t \leq T, x \in \mathbb{R},
$$

where $\varphi$ is the function in (2), and the simple-layer potential

$$
u_{1}(s, x, t)=\int_{s}^{t} G(s, x, \tau, X(\tau)) V(\tau, t) d \tau, 0 \leq s<t \leq T, x \in \mathbb{R},
$$

with density $V$ which is continuous in $s \in[0, t)$ and satisfies the inequality

$$
|V(s, t)| \leq c(t-s)^{-1+\varepsilon}
$$

for any $\varepsilon>0$. The last inequality ensures the validity of the formula on the jump for potential $u_{1}($ see $[9, \mathrm{Ch} . \mathrm{V}, \S \S 2-4])$

$$
D_{x}^{1} u_{1}(s, X(s), t)=-\frac{V(s, t)}{b(s, X(s))}+\int_{s}^{t} D_{x}^{1} G(s, X(s), \tau, X(\tau)) V(\tau, t) d \tau, 0 \leq s<t \leq T,
$$

where

$$
\begin{aligned}
D_{x}^{1} G(s, X(s), \tau, X(\tau))= & \frac{X(\tau)-X(s)}{b(\tau, X(\tau))(\tau-s) \sqrt{2 \pi b(\tau, X(\tau))(\tau-s)}} e^{-\frac{(X(\tau)-X(s))^{2}}{2 b(\tau, X(\tau))(\tau-s)}}+ \\
& +D_{x}^{1} Z_{1}(s, X(s), \tau, X(\tau)) .
\end{aligned}
$$

Furthermore, from condition 3 and estimate (6) it follows that

$$
\left|D_{x}^{1} G(s, X(s), \tau, X(\tau))\right| \leq c(\tau-s)^{-1+\frac{\alpha}{2}}, 0 \leq s<t \leq T .
$$

We find the solution of problem (1)-(3) of the form

$$
u(s, x, t)=u_{0}(s, x, t)+u_{1}(s, x, t), 0 \leq s<t \leq T, x \in \bar{D}_{s},
$$

with the unknown function $V$ to be determined.

If we substitute the expression (9) for $u(s, x, t)$ into (3), we obtain, upon using the relation (7), the following Volterra integral equation of the second kind

$$
V(s, t)=\int_{s}^{t} K(s, \tau) V(\tau, t) d \tau+\Psi(s, t), 0 \leq s<t \leq T,
$$


where

$$
\begin{aligned}
& \Psi(s, t)=b(s, X(s))\left(D_{x}^{1} u_{0}(s, X(s), t)+\frac{1}{q(s)} \int_{D_{s}}\left(u_{0}(s, y, t)-u_{0}(s, X(s), t)\right) \mu(s, d y)\right), \\
& K(s, \tau)=b(s, X(s)) \times \\
& \times\left(D_{x}^{1} G(s, X(s), \tau, X(\tau))+\frac{1}{q(s)} \int_{D_{s}}(G(s, y, \tau, X(\tau))-G(s, X(s), \tau, X(\tau))) \mu(s, d y)\right) .
\end{aligned}
$$

In order to solve this integral equation, we have to study the behavior of function $\Psi$ and kernel $K$. We begin with estimate for $\Psi$. Write $\Psi(s, t)$ in the form

$$
\begin{aligned}
\Psi(s, t)= & b(s, X(s))\left(D_{x}^{1} u_{0}\left(s, X_{i}(s), t\right)+\frac{1}{q(s)} \int_{D_{\delta}(X(s))}\left(u_{0}(s, y, t)-u_{0}(s, X(s), t)\right) \mu(s, d y)-\right. \\
& \left.+\frac{1}{q(s)} \int_{D_{s} \backslash D_{\delta}(X(s))}\left(u_{0}(s, y, t)-u_{0}(s, X(s), t)\right) \mu(s, d y)\right)= \\
& =b(s, X(s))\left(\Psi_{1}(s, t)+\Psi_{2}(s, t)+\Psi_{3}(s, t)\right) .
\end{aligned}
$$

For function $\Psi_{1}(s, t)$ we have

$$
\left|\Psi_{1}(s, t)\right|=\left|D_{x}^{1} u_{0}(s, X(s), t)\right| \leq c\|\varphi\|(t-s)^{-\frac{1}{2}} .
$$

To estimate $\Psi_{2}(s, t)$, apply the Lagrange formula to the integrand $u_{0}(s, y, t)-u_{0}(s, X(s), t)$ in its expression. We have

$$
u_{0}(s, y, t)-u_{0}(s, X(s), t)=D_{y}^{1} u_{0}(s, X(s)+\theta(y-X(s)), t) \cdot(y-X(s)),
$$

where $\theta$ is some real number from interval $(0,1)$. Hence

$$
\begin{aligned}
& \left|\Psi_{2}(s, t)\right|=\left|\frac{1}{q(s)} \int_{D_{\delta}(X(s))}\left(u_{0}(s, y, t)-u_{0}(s, X(s), t)\right) \mu(s, d y)\right|= \\
& =\left|\frac{1}{q(s)} \int_{D_{\delta}(X(s))} D_{y}^{1} u_{0}(s, X(s)+\theta(y-X(s)), t) \cdot(y-X(s)) \mu(s, d y)\right| \leq \\
& \leq c(\delta)\|\varphi\|(t-s)^{-\frac{1}{2}} .
\end{aligned}
$$

The same estimate is also valid for $\Psi_{3}(s, t)$. Indeed, using the triangle inequality, we obtain 


$$
\begin{aligned}
& \left|\Psi_{3}(s, t)\right|=\left|\frac{1}{q(s)} \int_{D_{s} \backslash D_{\delta}(X(s))}\left(u_{0}(s, y, t)-u_{0}(s, X(s), t)\right) \mu(s, d y)\right| \leq \\
& \quad \leq \frac{1}{q(s)} \int_{D_{s} \backslash D_{\delta}(X(s))}\left(\left|u_{0}(s, y, t)\right|+\left|u_{0}(s, X(s), t)\right|\right) \mu(s, d y) \leq c(\delta)\|\varphi\|(t-s)^{-\frac{1}{2}} .
\end{aligned}
$$

Combining (11), (12) and (13), we conclude that

$$
|\Psi(s, t)| \leq c_{0}(\delta)\|\varphi\|(t-s)^{-\frac{1}{2}} .
$$

where $c_{0}(\delta)$ is some positive constant depending on $\delta$.

Now consider kernel $K(s, \tau)$. Write it as follows

$$
\begin{aligned}
& K(s, \tau)=b(s, X(s)) \times \\
& \times\left[D_{x}^{1} G(s, X(s), \tau, X(\tau))+\frac{1}{q(s)} \int_{D_{s} \backslash D_{\delta}(X(s))}(G(s, y, \tau, X(\tau))-G(s, X(s), \tau, X(\tau))) \mu(s, d y)\right. \\
& +\frac{1}{q(s)} \int_{D_{\delta}(X(s))}\left(Z_{0}(s, y, \tau, X(\tau))-Z_{0}(s, X(s), \tau, X(\tau))\right) \mu(s, d y)+ \\
& \left.+\frac{1}{q(s)} \int_{D_{\delta}(X(s))}\left(Z_{1}(s, y, \tau, X(\tau))-Z_{1}(s, X(s), \tau, X(\tau))\right) \mu(s, d y)\right] .
\end{aligned}
$$

The first term in square brackets in the above expression is already estimated by (8). The absolute values of the second and fourth terms are bounded, respectively, by

$$
c(\delta)(\tau-s)^{-\frac{1}{2}} \text { and } c(\delta)(\tau-s)^{-\frac{1+\alpha}{2}}
$$

which becomes clear, respectively, after using the inequality (4) with $r=0, p=0$ and after applying the Lagrange formula to difference $Z_{1}(s, y, \tau, X(\tau))-Z_{1}(s, X(s), \tau, X(\tau))$ and using the inequality (6) with $r=0, p=1$, successively.

It remains to estimate the third term in the expression for $K(s, \tau)$ which we denote by $R(s, \tau)$. Write it in the form

$$
\begin{aligned}
& R(s, \tau)=\frac{1}{q(s) \sqrt{2 \pi b(\tau, X(\tau))(\tau-s)}} \int_{D_{\delta}(X(s))}\left(e^{-\frac{(y-X(\tau))^{2}}{2 b(\tau, X(\tau))(\tau-s)}}-e^{-\frac{(X(\tau)-X(s))^{2}}{2 b(\tau, X(\tau))(\tau-s)}}\right) \mu(s, d y)= \\
& =-\frac{1}{q(s) \sqrt{2 \pi b(\tau, X(\tau))(\tau-s)}} \int_{D_{\delta}(X(s))} \mu(s, d y) \int_{0}^{1} \frac{\partial}{\partial \theta}\left(e^{-(1-\theta) \frac{(y-X(\tau))^{2}}{2 b(\tau, X(\tau))(\tau-s)}}-e^{-\theta \frac{(X(s)-X(\tau))^{2}}{2 b(\tau, X(\tau))(\tau-s)}}\right) d \theta .
\end{aligned}
$$


Taking the derivative $\frac{\partial}{\partial \theta}\left(e^{-(1-\theta) \frac{(y-X(\tau))^{2}}{2 b(\tau, X(\tau))(\tau-s)}}-e^{-\theta \frac{(X(s)-X(\tau))^{2}}{2 b(\tau, X(\tau))(\tau-s)}}\right)$ and then using the equality $(y-X(\tau))^{2}-(X(\tau)-X(s))^{2}=(y-X(s))^{2}-2(X(\tau)-X(s))(y-X(s))$, we get

$$
\begin{aligned}
R(s, \tau) & =\frac{X(\tau)-X(s)}{q(s) b(\tau, X(\tau))(\tau-s) \sqrt{2 \pi b(\tau, X(\tau))(\tau-s)}} \times \\
& \times \int_{0}^{1} d \theta \int_{D_{\delta}(X(s))}(y-X(s))\left(e^{-(1-\theta) \frac{(y-X(\tau))^{2}}{2 b(\tau, X(\tau))(\tau-s)}}-e^{-\theta \frac{(X(s)-X(\tau))^{2}}{2 b(\tau, X(\tau))(\tau-s)}}\right) \mu(s, d y)- \\
& -\frac{1}{2 q(s) b(\tau, X(\tau))(\tau-s) \sqrt{2 \pi b(\tau, X(\tau))(\tau-s)}} \times \\
& \times \int_{0}^{1} d \theta \int_{D_{\delta}(X(s))}(y-X(s))^{2}\left(e^{-(1-\theta) \frac{(y-X(\tau))^{2}}{2 b(\tau, X(\tau))(\tau-s)}}-e^{-\theta \frac{(X(s)-X(\tau))^{2}}{2 b(\tau, X(\tau))(\tau-s)}}\right) \mu(s, d y)= \\
& =R_{1}(s, \tau)+R_{2}(s, \tau) .
\end{aligned}
$$

From condition 3 it follows that

$$
\left|R_{1}(s, \tau)\right| \leq c(\tau-s)^{-\frac{1+\alpha}{2}} \int_{0}^{1} d \theta \int_{D_{\delta}(X(s))}(y-X(s)) \mu(s, d y) \leq c(\delta)(\tau-s)^{-\frac{1+\alpha}{2}} .
$$

Consider $R_{2}(s, \tau)$. Since

we have

$$
\left|(1-\theta)(y-X(\tau))^{2}+\theta(X(s)-X(\tau))^{2}\right| \geq(1-\theta) \theta(y-X(s))^{2},
$$

$$
\begin{gathered}
\left|R_{2}(s, \tau)\right| \leq c(\tau-s)^{-\frac{3}{2}} \int_{0}^{1} d \theta \int_{D_{\delta}(X(s))}(y-X(s))^{2} e^{-\frac{(1-\theta) \theta}{2 b(\tau, X(\tau))(\tau-s)}(y-X(s))^{2}} \mu(s, d y) \leq \\
\leq c(\delta)(\tau-s)^{-1} \int_{0}^{1} \theta^{-\frac{1}{2}}(1-\theta)^{-\frac{1}{2}} d \theta \leq c(\delta)(\tau-s)^{-1} .
\end{gathered}
$$

Thus kernel $K(s, \tau)$ in (9) has strong singularity which is caused by $R_{2}(s, \tau)$. Therefore we do not know yet whether a solution of (10) exists. We shall see presently that it is nevertheless possible to obtain the solution of (10) by an ordinary method of successive approximations, i.e.,

where

$$
V(s, t)=\sum_{k=0}^{\infty} V^{(k)}(s, t)
$$

$$
\begin{aligned}
& V^{(0)}(s, t)=\Psi(s, t) \\
& V^{(k)}(s, t)=\int_{s}^{t} K(s, \tau) V^{(k-1)}(\tau, t) d \tau, k \in \mathbb{N} .
\end{aligned}
$$


Let us prove that the integrals on the right side of expression for $V_{k}(s, t)$ exist and the series (15) is convergent in $0 \leq s<t \leq T$. To do this, we first break the expression for $K(s, \tau)$ into two terms $K_{1}(s, \tau)$ satisfying the estimate (8) with some positive constant $c_{1}(\delta)$ and $K_{2}(s, \tau)=R_{2}(s, \tau)$ having strong singularity, i.e.,

$$
K(s, \tau)=K_{1}(s, \tau)+K_{2}(s, \tau), 0 \leq s<\tau<t \leq T .
$$

Next, consider $V^{(1)}(s, t)$ and represent it as follows

where

$$
V^{(1)}(s, t)=V_{1}^{(1)}(s, t)+V_{2}^{(1)}(s, t)
$$

$$
V_{i}^{(1)}(s, t)=\int_{s}^{t} K_{i}(s, \tau) \Psi(\tau, t) d \tau, i=1,2 .
$$

In view of estimate (8) (with constant $c_{1}(\delta)$ ) for $K_{1}(s, \tau)$ and inequality (14), we immediately deduce that

$$
\begin{aligned}
\left|V_{1}^{(1)}(s, t)\right| & \leq c_{0}(\delta) c_{1}(\delta)\|\varphi\| \int_{s}^{t}(\tau-s)^{-1+\frac{\alpha}{2}}(t-\tau)^{-\frac{1}{2}} d \tau \leq \\
& \leq c_{0}(\delta) c_{1}(\delta)\|\varphi\| \frac{\Gamma\left(\frac{\alpha}{2}\right) \Gamma\left(\frac{1}{2}\right)}{\Gamma\left(\frac{1+\alpha}{2}\right)}(t-s)^{-\frac{1}{2}+\frac{\alpha}{2}} .
\end{aligned}
$$

To estimate $V_{2}^{(1)}$, write

$\left|V_{2}^{(1)}(s, t)\right|=\left|\int_{s}^{t} K_{2}(s, \tau) \Psi(\tau, t) d \tau\right|=\frac{1}{2 q(s) \sqrt{2 \pi}} \times$

$\times\left|\int_{s}^{t} \Psi(\tau, t) d \tau \int_{0}^{1} d \theta \int_{D_{\delta}(X(s))} \frac{(y-X(s))^{2} \mu(s, d y)}{(b(\tau, X(\tau))(\tau-s))^{\frac{3}{2}}}\left(e^{-(1-\theta) \frac{(y-X(\tau))^{2}}{2 b(\tau, X(\tau))(\tau-s)}}-e^{-\theta \frac{(X(s)-X(\tau))^{2}}{2 b(\tau, X(\tau))(\tau-s)}}\right)\right| \leq$

$\leq \frac{c_{0}(\delta)\|\varphi\|}{2 b q(s) \sqrt{2 \pi b}} \int_{0}^{1} d \theta \int_{D_{\delta}(X(s))}(y-X(s))^{2} \mu(s, d y) \int_{s}^{t}(t-\tau)^{-\frac{1}{2}}(\tau-s)^{-\frac{3}{2}} e^{-\theta(1-\theta) \frac{(y-X(s))^{2}}{2 B \cdot(\tau-s)}} d \tau$,

where $b$ and $B$ are constants in 1 .

Denote by $I(s, \tau)$ the inner integral in the last relation. Write it in the form

$$
\begin{aligned}
I(s, \tau) & =\int_{s}^{t}(t-\tau)^{-\frac{1}{2}}(\tau-s)^{-\frac{3}{2}} e^{-\theta(1-\theta) \frac{(y-X(s))^{2}}{2 B \cdot(\tau-s)}} d \tau= \\
& =e^{-\theta(1-\theta) \frac{(y-X(s))^{2}}{2 B \cdot(t-s)}} \int_{s}^{t}(t-\tau)^{-\frac{1}{2}}(\tau-s)^{-\frac{3}{2}} e^{-\theta(1-\theta) \frac{(y-X(s))^{2}}{2 B \cdot(t-s)} \frac{t-\tau}{\tau-s}} d \tau
\end{aligned}
$$


Changing the variable of integration $\tau$ into $z=\frac{t-\tau}{\tau-s}$, we obtain

$$
\begin{aligned}
I(s, \tau) & =e^{-\theta(1-\theta) \frac{(y-X(s))^{2}}{2 B \cdot(t-s)}} \frac{1}{t-s} \int_{0}^{\infty} z^{-\frac{1}{2}} e^{-\theta(1-\theta) \frac{(y-X(s))^{2}}{2 B \cdot(t-s)} z} d z= \\
& =\sqrt{\frac{2 \pi B}{(t-s) \theta(1-\theta)}} \frac{1}{(y-X(s))} e^{-\theta(1-\theta) \frac{(y-X(s))^{2}}{2 B \cdot(t-s)}} .
\end{aligned}
$$

In view of (18), we get

$$
\left|V_{2}^{(1)}(s, t)\right| \leq c_{0}(\delta) \lambda_{s}(\delta)\|\varphi\|(t-s)^{-\frac{1}{2}}
$$

where

$$
\lambda_{s}(\delta)=\frac{\pi}{2 q(s)} \sqrt{\frac{B}{b}} \int_{D_{\delta}(X(s))}(y-X(s)) \mu(s, d y) .
$$

Combining (17) and (19), we conclude that

$$
\left|V^{(1)}(s, t)\right| \leq c_{0}(\delta)\|\varphi\|(t-s)^{-\frac{1}{2}}\left[\frac{c_{1}(\delta) \Gamma\left(\frac{\alpha}{2}\right) \Gamma\left(\frac{1}{2}\right)}{\Gamma\left(\frac{1+\alpha}{2}\right)}(t-s)^{\frac{\alpha}{2}}+\lambda_{s}(\delta)\right],
$$

Choose $\delta=\delta_{0}$ so small that $\lambda_{s}\left(\delta_{0}\right)<1$ and denote

$$
\lambda_{s}=\lambda_{s}\left(\delta_{0}\right), c_{0}=c_{0}\left(\delta_{0}\right), c_{1}=c_{1}\left(\delta_{0}\right) .
$$

Proceeding by induction, we derive the following estimates for terms $V^{(k)}(s, t)$ of series (15)

$$
\left|V^{(k)}(s, t)\right| \leq c_{0}\|\varphi\|(t-s)^{-\frac{1}{2}} \sum_{n=0}^{k}\left(\begin{array}{l}
k \\
n
\end{array}\right) h_{s, t}^{(k-n)}\left(\lambda_{s}\right)^{n}, k=0,1,2, \ldots
$$

where

$$
h_{s, t}^{(n)}=\frac{\left[c_{1} \Gamma\left(\frac{\alpha}{2}\right)\right]^{n} \Gamma\left(\frac{1}{2}\right)}{\Gamma\left(\frac{1+n \alpha}{2}\right)}(t-s)^{n \cdot \frac{\alpha}{2}}, n=0,1,2, \ldots
$$


Hence, for $0 \leq s<t \leq T$, we have

$$
\begin{aligned}
\left|\sum_{k=0}^{\infty} V^{(k)}(s, t)\right| & \leq c_{0}\|\varphi\|(t-s)^{-\frac{1}{2}} \sum_{k=0}^{\infty} \sum_{n=0}^{k}\left(\begin{array}{l}
k \\
n
\end{array}\right) h_{s, t}^{(k-n)}\left(\lambda_{s}\right)^{n}= \\
& =c_{0}\|\varphi\|(t-s)^{-\frac{1}{2}} \sum_{k=0}^{\infty} h_{s, t}^{(k)} \sum_{n=0}^{k}\left(\begin{array}{c}
n+k \\
n
\end{array}\right)\left(\lambda_{s}\right)^{n}= \\
& =c_{0}\|\varphi\|(t-s)^{-\frac{1}{2}} \sum_{k=0}^{\infty} \frac{h_{s, t}^{(k)}}{\left(1-\lambda_{s}\right)^{k+1}} .
\end{aligned}
$$

This implies that series (15) is absolutely convergent in $0 \leq s<t \leq T$ and therefore the function $V(s, t)$ exists and satisfies the inequality

$$
|V(s, t)| \leq c\|\varphi\|(t-s)^{-\frac{1}{2}}, 0 \leq s<t \leq T .
$$

We have thus constructed a solution $u(s, x, t)$ of the boundary-value problem (1)-(3) of the form (9). From relations (4)-(6) and (21) it follows that

$$
u \in C^{1,2}\left(S_{t}\right) \cap C\left(\overline{S_{t}}\right)
$$

and

$$
|u(s, x, t)| \leq c\|\varphi\|
$$

The proof of uniqueness of solution of (1)-(3) is based on the maximum principle for parabolic equations and is a repetition of the proof of the analogous assertion in [3] with obvious changes.

We have proved the following theorem:

Theorem 1. Let the conditions 1-6 hold. Then the problem (1)-(3) has a unique solution $u(s, x, t)$ in $C^{1,2}\left(S_{t}\right) \cap C\left(\bar{S}_{t}\right)$. Furthermore, this solution has the form (9) and satisfies the estimate (22).

\section{Construction of the Feller semigroup}

Consider the following problem: construct the two-parameter semigroup of operators $T_{s, t}, 0 \leq s<t \leq T$, which describes the inhomogeneous Feller process on $\bar{D}_{s} \equiv[X(s), \infty)$ connected with (1)-(3). Such a Feller process coincides in $D_{s}$ with the diffusion process given by (1), (2) (with drift $a(s, x)$ and diffusion coefficient $b(s, x)$ ) and its behavior at boundary point $X(s)$ is determined by the Feller-Wentzell boundary condition (3). Note that the two terms of boundary condition (3) 


$$
-q(s) \frac{\partial u(s, X(s), t)}{\partial x} \text { and } \int_{D_{s}}(u(s, X(s), t)-u(s, y, t)) \mu(s, d y)
$$

are supposed to correspond to the reflection phenomenon and the jump phenomenon on the boundary $X(s)$.

We introduce the two-parameter family of linear operators $T_{s, t}, 0 \leq s<t \leq T$, acting on the space $C_{b}(\mathbb{R})$ by the rule

$$
T_{s, t} \varphi(x)=u(s, x, t, \varphi)
$$

where $u(s, x, t, \varphi) \equiv u(s, x, t)$ is the solution of problem (1)-(3) defined by formulas (9), (15).

Let us show that the family of operators $T_{s, t}$ is the desired semigroup. To do this, we first note that the operators $T_{s, t}$ have the following property: if the sequence $\varphi_{n} \in C_{b}(\mathbb{R})$ is such that $\lim _{n \rightarrow \infty} \varphi_{n}(x)=\varphi(x)$ for all $x \in \bar{D}_{s}$ and $\sup _{n}\left\|\varphi_{n}\right\|<\infty$, then $\lim _{n \rightarrow \infty} T_{s, t} \varphi_{n}(x)=T_{s, t} \varphi(x)$ for all $0 \leq s<t \leq T, x \in \bar{D}_{s}$. The proof of this property is based on well-known assertions of calculus on the passage of the limit under the summation and integral signs (here this concerns series (15) and integrals on the right side of the expression (9)). This property allows us to prove the next properties of the operator family $T_{s, t}$, without loss of generality, under the assumption that the function $\varphi$ has a compact support.

The next lemma asserts that the operators $T_{s, t}$ are positivity preserving:

Lemma 1. If $\varphi \in C_{b}(\mathbb{R})$ and $\varphi(x) \geq 0$ for all $x \in \bar{D}_{s}$, then $T_{s, t} \varphi(x) \geq 0$ for all $0 \leq s<t \leq T, x \in \bar{D}_{s}$.

Proof. Let $\varphi$ be any nonnegative function in $C_{b}(\mathbb{R})$ having compact support. Denote by $\gamma$ the minimum of $T_{s, t} \varphi(x)$ in $(s, x) \in[0, t] \times \bar{D}_{s}$ and assume that $\gamma<0$. From the minimum principle it follows that there exists $s_{0} \in(0, t)$ such that $T_{s_{0}, t} \varphi\left(X\left(s_{0}\right)\right)=\gamma$. But then the inequalities

$$
\frac{\partial T_{s_{0}, t} \varphi\left(X\left(s_{0}\right)\right)}{\partial x} \geq 0 \text { and } \int_{D_{s}}\left(T_{s_{0}, t} \varphi\left(X\left(s_{0}\right)\right)-T_{s_{0}, t} \varphi(y)\right) \mu(s, d y) \leq 0
$$

hold. Furthermore, Theorem 14 in $[9$, p. 69] assures us that

$$
\frac{\partial T_{s_{0}, t} \varphi\left(X\left(s_{0}\right)\right)}{\partial x}>0 \text {. }
$$

Next, since $q\left(s_{0}\right)>0$, it becomes clear that the fulfillment of condition (3) is impossible. The contradiction we arrived at indicates that $\gamma \geq 0$. This completes the proof of the lemma. 
Another important property of operators $T_{s, t}$ is that they are contractive, i.e.,

$$
\left\|T_{s, t} \varphi\right\| \leq\|\varphi\|, 0 \leq s<t \leq T .
$$

This property follows from Lemma 1 together with the fact that if $\varphi_{0}(x) \equiv 1$ then $T_{s, t} \varphi_{0}(x)=1$ for all $0 \leq s<t \leq T, x \in \bar{D}_{s}$.

Finally, we show that operator family $T_{s, t}$ has the semigroup property

$$
T_{s, t}=T_{s, \tau} T_{\tau, t}, 0 \leq s<\tau<t \leq T .
$$

This property is a consequence of the assertion of uniqueness of the solution of the problem (1)-(3). Indeed, to find $u(s, x, t)$ when $\lim _{s \uparrow t} u(s, x, t)=\varphi(x)$, we can solve the problem (1)-(3) first in the time interval $[\tau, t]$ with the "initial" function $\varphi(x)$, and then in the time interval $[s, \tau]$ with the "initial" function $T_{\tau, t} \varphi(x)$. In other words, $T_{s, t} \varphi(x)=T_{s, \tau}\left(T_{\tau, t} \varphi\right)(x), \varphi \in C_{b}(\mathbb{R})$, or $T_{s, t}=T_{s, \tau} T_{\tau, t}$.

The above properties of operators $T_{s, t}$ imply the following assertion (see [10, Ch. II], §1):

Theorem 2. Let the conditions of Theorem 1 hold. Then the two parameter semigroup of operators $T_{s, t}, 0 \leq s<\tau<t \leq T$, defined by (23) describes the inhomogeneous Feller process on $\bar{D}_{s}$ which coincides in $D_{s}$ with the diffusion process given by (1), (2) and its behavior at point $X(s)$ is determined by the Feller-Wentzell boundary condition (3).

\section{References}

[1] Feller W., The parabolic differential equations and associated semi-groups of transformations, Ann. Math. 1952, 55, 468-518.

[2] Wentzell A.D., Semigroups of operators that correspond to a generalized differential operator of second order, Dokl. AN SSSR 1956, 111(2), 269-272 (in Russian).

[3] Shevchuk R.V., Inhomogeneous diffusion processes on a half-line, generated by the differential operator with Feller-Wentzell boundary condition, Math. Bull. NTSH 2011, 8, 243-257 (in Ukrainian).

[4] Kopytko B.I., Shevchuk R.V., Diffusions in one-dimensional bounded domains with reflection, absorption and jumps at the boundary and at some interior point, Journal of Applied Mathematics and Computational Mechanics 2013, 12(1), 55-68.

[5] Portenko M.I., Diffusion Processes in Media with Membranes, Institute of Mathematics of the NAS of Ukraine, Kyiv 1995 (in Ukrainian).

[6] Pilipenko A.Yu., On the Skorokhod mapping for equations with reflection and possible jump-like exit from a boundary, Ukrainian Math. J. 2012, 63(9), 1415-1432.

[7] Anulova S.V., On stochastic differential equations with boundary conditions in a half-plane, Izv. AN SSSR Ser. Mat. 1981, 45(3), 491-508 (in Russian).

[8] Ladyzhenskaya O.A., Solonnikov V.A., Ural'tseva N.N., Linear and Quasilinear Equations of Parabolic Type, Nauka, Moscow 1967 (in Russian).

[9] Friedman A., Partial Differential Equations of Parabolic Type, Mir, Moscow 1968 (in Russian).

[10] Dynkin E.B., Markov Processes, Fizmatgiz, Moscow 1963 (in Russian). 